\title{
Surgical Excision of Oral Verrucose Carcinoma Using Diode Laser: A Case Report
}

\section{Sibgatullah' ${ }^{1}$, Jayesh ${ }^{2 *}$, Shreedevi Bhoi ${ }^{3}$, Aklanta Kumar Gogoi ${ }^{4}$, Arundhati Singh ${ }^{2}$ and Vidhya Ningrajappa ${ }^{4}$}

${ }^{1}$ Director, M.K Advanced Dental Hospital, Ranchi, India

${ }^{2}$ Senior Lecturer, Department of Oral and Maxillofacial Surgery, Hazaribagh

College of Dental Sciences, India

${ }^{1}$ Reader, Department of Oral and Maxillofacial Surgery, Hazaribagh College of

Dental Sciences, India

${ }^{4}$ M.D.S Oral and Maxillofacial Surgery, India

*Corresponding Author: Jayesh, Senior Lecturer, Department of Oral and

Maxillofacial Surgery, Hazaribagh College of Dental Sciences, India.
Received: September 14, 2020

Published: September 25, 2020

(C) All rights are reserved by Jayesh., et al.

\begin{abstract}
Background: Oral Verrucous Carcinoma is a form of oral cancer with a very less malignant potency. There are variable number of verrucous tumors of oral cavity which are hard to distinguish clinically. It is a slow growing tumor and which rarely metastasize. The tumor is mostly seen on the buccal mucosa and is associated with tobacco and similar habits. The treatment of choice is surgical excision with wide margins. We are presenting a case of Oral Verrucous Carcinoma who reported to our department. The lesion was slow growing and present for the past 6 months. Incisional biopsy was followed by excision of the lesion using Diode laser ( $980 \mathrm{~nm}$ at 2 watts and at pulse mode). The procedure was uneventful and patient was discharged from the outpatient. Post-surgical follow up was normal with minimal pain and edema.
\end{abstract}

Conclusion: Oral Verrucous Carcinoma is a tumor with low metastatic potency. The use of Diode laser for excision of the tumor with follow up is a good treatment of choice for moderate sized tumors. With the clear field and less post-operative complications Diode laser is good instrument in the hands of a surgeons.

Keyword: Oral Verrucous Carcinoma; Diode Laser; Biopsy; Dysplasia; Metastasis; Cancer

\section{Introduction}

Oral Verrucous Carcinoma (OVC) is a rare tumor which was first described by Ackerman [1]. It is a special form of well-differentiated squamous cell carcinoma having specific clinical and histological features [1]. The tumor grows slowly, which is locally invasive in nature and unlikely to metastasize. It appears as a painless, thick white plaque resembling a cauliflower. The most common sites of oral mucosal involvement include the buccal mucosa, followed by the mandibular alveolar crest, gingiva, and tongue. Surgery has been the first choice of treatment for these lesions while manage- ment with radiotherapy is controversial; however, surgery combined with radiotherapy is the next most preferable treatment with benefits, particularly in cases of extensive lesions [1].

The management of oral verrucous carcinoma is rarely radical or multi-modal. Even with local tumor aggression, it is intriguing that regional or distant metastasis is rare. Despite these differences, most guidelines and treatment protocols do not differentiate between OVC and OSCC with respect to the margins of excision, need for adjuvant therapy, or the role and extent of lymph node dissection [2]. 


\section{Case Report}

A 38 year old female patient had visited the Department of oral and maxillofacial surgery with a chief complain of a growth in her left cheek region for 6 months (Figure 1). The patient gave a history of approaching a clinic with same complain two months earlier. The patient didn't undergo further treatment because of cost. A history of betel nut chewing for 15 years was elicited. On examination a single left submandibular lymph node was palpated which was non-tender and mobile. Following routine blood investigations incisional biopsy of the lesion was advised and a provisional diagnosis of Verrucous hyperplasia was rendered. A decision to excise the lesion with diode laser was undertaken under local anesthesia.

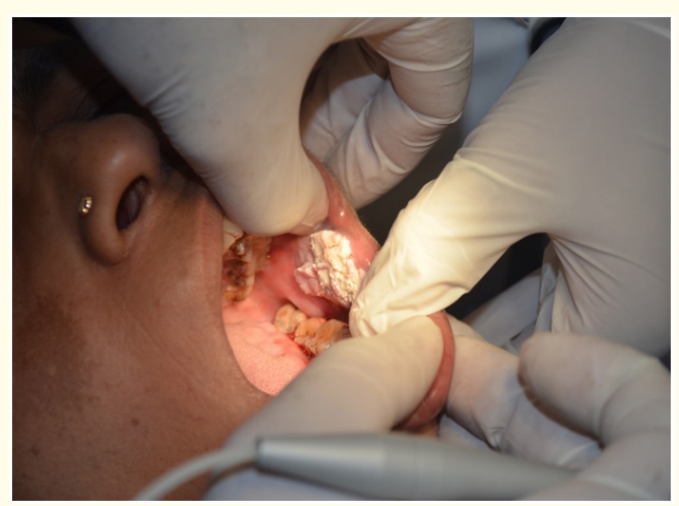

Figure 1: Patient presented with a growth on the left buccal mucosa.

A betadine skin scrub and intra oral betadine wash was done before surgical procedure. Long buccal nerve block was given on the left side with a local anesthetic agent (2\% lignocaine with 1:200000 adrenaline). Infiltration was also given along the margins of the growth. Procedure was started after obtaining anesthesia and wearing of laser protecting eye wear. A $980 \mathrm{~nm}$ (Doctor smile) soft tissue laser was used for the procedure. The laser was used in pulse mode as it reduces the thermal effects of the laser. Activated laser tip was used for giving the incision at maximum wattage of 2 watts. To achieve greater depth the laser was passed through the area several times. An initial margin of $1 \mathrm{~cm}$ was given all around the growth. Once the margin was obtained the laser was passed through the area to achieve the desired depth. On reaching a safe depth the tumor was undermined using the laser (Figure 2). Angular artery was observed while dissection was going on. The artery was protected by retraction.

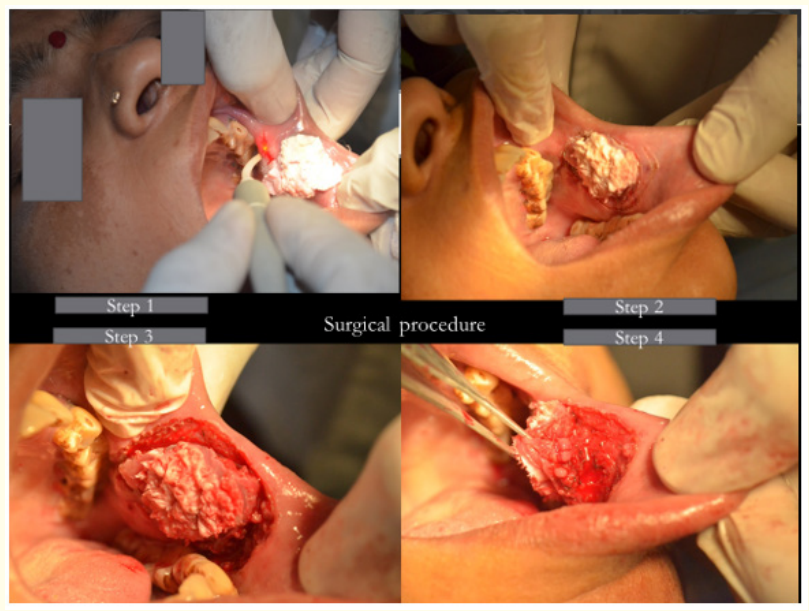

Figure 2: Steps in the surgical excision. Step 1- Diode laser is used to make margin away from the lesion. Step 2- A well defined margin is made all around the lesion. Step 3- The initial incision is deepened. Step 4. The lesion is undermined using laser.

Following the dissection, and identifying the margins, tumor was removed and sent for histopathological evaluation. The excision of the lesion created a defect of about $4 \mathrm{X} 4 \mathrm{~cm}$ (Figure 3). Primary closure of the defect was done with simple interrupted suturing using 3-0 Vicryl suture. Following suturing the patient was given I.M pain killers. The patient was kept under observation for an hour for any post-operative complication. Patient was discharged after verifying there was no bleeding from the surgical site. Antibiotics and analgesics were prescribed. Patient was then followed up on $3^{\text {rd }}$ and $5^{\text {th }}$ day (Figure 4).

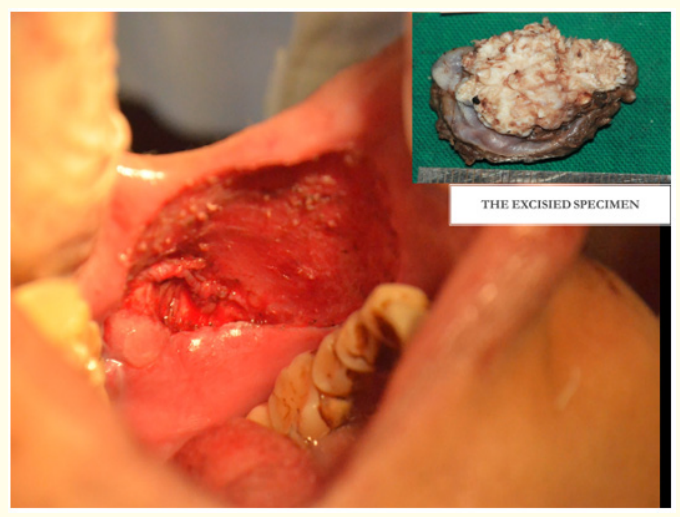

Figure 3: Mucosa after excision of lesion. Right top corner offset shows the excised specimen. Angular artery is seen in the left corner. 


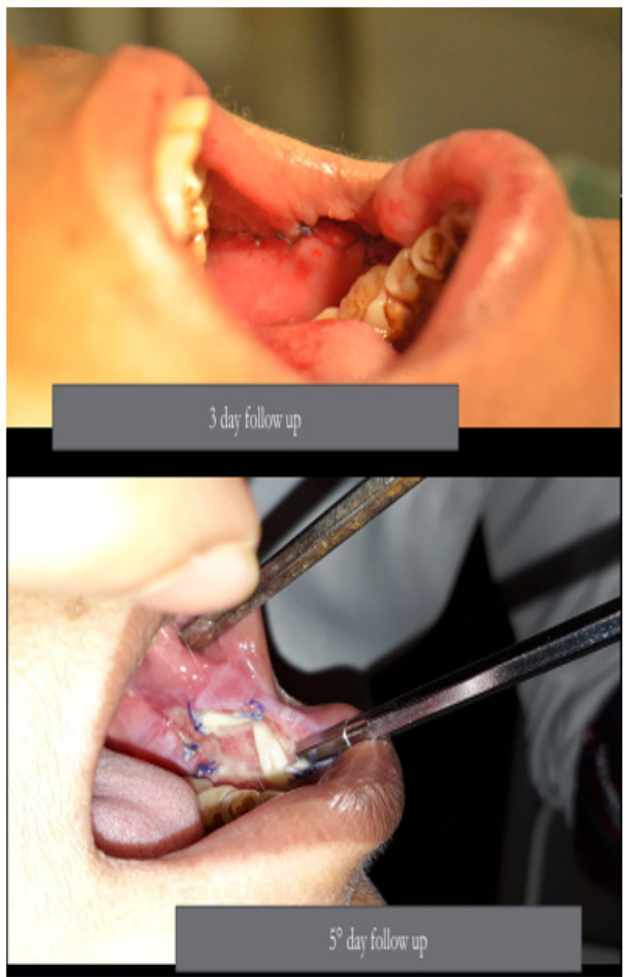

Figure 4: Follow up. Upper pic 3rd day intra oral wound healing. Lower pic 5th day follow up.

Patient had minimum edema, pain and the mouth opening was found to be adequate. The biopsy result showed the tumor as verrucous carcinoma. The margins were free from any dysplastic changes. Patient was further followed up for a period of one year, with no recurrence noted during that period.

\section{Discussion}

Schrader, et al. and Jordan have reported that verrucous carcinomas are slow-growing, exophytic, well-demarcated hyperkeratotic lesions which typically presents as extensive, white, warty lesions [1]. The etiology of verrucous carcinoma is not well defined. Human papillomavirus (HPV) has been considered as one of the causative factors. Smoking in form of beedies, smokeless tobacco, chewing tobacco along with poor oral hygiene and oral lichen planus are also considered as predisposing factor for oral verrucous carcinoma [3].

Verrucous surface may belong to a broad spectrum of diseases ranging from Verrucous hyperplasia, pseudo epithelial hyperpla- sia, proliferative verrucous leukoplakia to hybrid Oral verrucous carcinoma [2]. It is clinically difficult to diagnose these entities differently although on histological examination verrucous hyperplasia is superficially than carcinoma which extends deeper [3]. Oral verrucous carcinoma has a Para keratinized epithelium with irregular clefted surface and parakeratin plugging extending deep into clefts. The tumor has well defined basement membrane with no discontinuity with minimal cellular changes and sub epithelial inflammation [3].

Surgical removal of the tumor with wide margins is adequate in management of oral verrucous carcinoma [2]. Prognosis is better than other forms of oral cancers with irradiation rarely needed except in cases where tumor extends till retro molar area when combination therapy is beneficial [3].

\section{LASERS as a treatment modality for verrucous carcinoma}

Yeh C J in a study showed shaving and cryosurgery as an effective treatment modality for Verrucous Carcinoma/hyperplasia. The authors also noted that laser surgery can be a good treatment option for small to medium lesions. Only drawback being the equipment not readily available [4].

Theodore Maiman created the first "laser" (light amplification by stimulated emission of radiation) by using an electrical source to energize a solid ruby. Various types of lasers currently being used for medical and surgical procedures are $\mathrm{CO}_{2}$ lasers, Diode Lasers, Nd-Yag etc [5].

Direct laser ablation has been used for direct destruction of cancer cells through its photochemical, photomechanical, and photothermal effects. The photochemical reactions that occur ultimately forms toxic radicals that lead to the death of tissues, the photomechanical reactions induce stress on the tissue and lead to its fragmentation and the photothermal reactions induce heating and coagulation, which cause cell death.

In our case a $980 \mathrm{~nm}$ Diode Laser was used (wavelength of diode lasers varies from 810 - $1064 \mathrm{~nm}$ ). Laser light at 800 - 980 $\mathrm{nm}$ is poorly absorbed in water, but highly absorbed in hemoglobin and other pigments. The hard tissues don't absorb this laser light. Hence it makes the dental diode laser very useful for soft tissue surgical procedures in oral cavity. These properties gives a diode laser very good efficiency for cutting gingiva and other oral mucosa which are highly vascularized and pigmented. The diode laser 
tissue penetration is also less than Nd -YaG lasers so the cutting is more controlled. One of the biggest advantages of using any laser is the bloodless field as it cauterizes the blood vessels $[5,6]$.

In our case we saw all the advantages of using a diode laser in the oral cavity. The laser took longer time than a Scalpel blade would have taken but this drawback was offset by relatively clean field of surgery and minimal blood loss from the surgical site during procedure. Patient was discharged from the OPD after an hour of observation. In the follow up the patient's mouth opening was adequate with no compromise on functioning namely speech and mastication process. The wound healed uneventfully with minimal scarring and post-operative edema.

\section{Conclusion}

Verrucous Carcinoma of the oral cavity is a slow growing tumor with very low metastatic potential. Diode laser can be used safely for excision of soft tissue lesions of the oral cavity. The use of a laser reduces the two most dreaded words for the patient which are pain and bleeding. The laser used is a simple instrument and when wisely used gives the surgeon a very versatile tool for surgical procedures in the oral cavity. With the patient getting more and more demanding use of lasers is a new weapon in the surgeon's arsenal.

\section{Source(S) of Support}

Nil.

\section{Conflicts of Interest}

Nil.

\section{Bibliography}

1. Alkan Bulut and Gunhan Ozden. "Oral Verrucous Carcinoma: A Study of 12 Cases”. European Journal of Dentistry 4.2 (2010): 202-207.

2. Franklyn J., et al. "Oral Verrucous Carcinoma: Ten Year Experience from a Tertiary Care Hospital in India". Indian Journal of Medical and Paediatric Oncology 38.4 (2017): 452-455.

3. Santosh HN., et al. "Verrucous Carcinoma: A clinicopathological study". Journal of Oral and Maxillofacial Pathology 23.2 (2019): 303.

4. CJ Yeh. "Treatment of Verrucous hyperplasia and Verrucous Carcinoma by shave excision and simple cryosurgery". International Journal of Oral and Maxillofacial Surgery 32.3 (2003): 280-283.
5. Beina Azadgoli and Regina Y Baker. "Laser applications in surgery”. Annals of Translational Medicine 4.23 (2016): 452.

6. Gupta G. "Management of Gingival Hyperpigmentation by Semiconductor Diode Laser". Journal of Cutaneous and Aesthetic Surgery 4.3 (2011): 208-210.

\section{Assets from publication with us}

- Prompt Acknowledgement after receiving the article

- Thorough Double blinded peer review

- Rapid Publication

- Issue of Publication Certificate

- High visibility of your Published work

Website: www.actascientific.com/

Submit Article: www.actascientific.com/submission.php

Email us: editor@actascientific.com

Contact us: +919182824667 